from the British troops in Cairo were not dissimilar during the months of 1886 and 1887 until October, when they rose from 17 to 7 per cent. In Norember, 1886 , the percentage was 1 per cent., and 6.4 in 1887 ; while in December, when the dengue epidemic was over, they were respectively 0.3 and 1 per cent. Possibly some of these cases in October and November were mild examples of dengue, in which the rash was absent or undiscovered. In Alexandria, where the epidemic was milder and of shorter duration, there was no perceptible difference between the simple continued fever admissions from the British troops in 1886 and 1887.

Varieties.-Dengue would seem to present varieties according to its degree of severity and the presence of certain symptoms or complications, such as persistent pain in particular joints, swelling of submaxillary glands, epistaxis, relapses, and the initial and terminal eruptions as seen in Calcutta in 1872. The chief features of the 1887 epidemic were the mildness and short duration of the symptoms, and the comparative absence of painand swelling in the joints.

Sequela. - There are in Egypt no complications of dengue, but the convalescence is curiously protracted, so that it may be weeks before the patient is able to resume all his ordinary occupations in consequence of his anzemia, debility, loss of flesh, and muscular pains. Vernoni in 1881 sug gested that after the epidemic of 1880 there were an un usual number of cases of inflammation and abscess of the liver, but this is not the experience of the present year. I have met with three cases of enteric fever, contracted while still feeble from an attack of dengue.

Prognosis and mortality. - With the exception of one case of malignant dengue at Port Said in 1883, I know of no fatal results in Egypt. One patient died of angina pectoris at the end of his convalescent period; but at Aden in 1871 a mortality of 5 out of 450 cases is reported by Nielly.

Treatment.-Quinine is apparently of no prophylactic value, and, as already stated, those who returned from Europe in perfect health were affected as easily as those who were exhausted by the damp heat of an unusually trying summer. There is no need for drug treatment in most cases. An emetic early in the attack seems to lower the temperature and benefit the malaise, and the bowels generally require to be gently purged. Quinine is of no arail to shorten the disease, but is useful as a general tonic when the tongue has cleaned during convalescence. Arsenic and other antimalarial drugs are also useless. Antipyrin and antifebrin in large doses will lower the temperature and the patient, but will not assuage pain or cut short the disease. Belladonna, recommended by one author for pain, I never found of any use. Salicylate of soda in doses of twenty grains seemed more useful for pain than other drugs. Chloral and bromide of potassium were used as sleeping draughts in bad cases. Tonics for the appetite and iron for anæmia are necessary for about a month during convalescence. Muscular rheumatism, when it persists, is benefited by antipyrin. During the febrile period, and for at least a fortnight afterwards, the patient will be unable to take solid food in any quantity, and will of course require nourishing liquids and a little wine. Cairo.

\section{A NEW FORM OF}

\section{INSTRUMENT FOR THE TREATMENT OF INFANTILE PARALYSIS INVOLVING THE EXTENSOR MUSCLES OF THE KNEE.}

BY W. J. WALSHAM, F.R.C.S.,

ASSISTANT SURGEON TO, AND SURGEON IN CHARGE OF, THE ORTHOPADIC DEPARTMENT AT ST. BARTHOLOMEW'S HOSPITAL

THERE is a class of cases, well known to those interested in orthopædic surgery, in which, in connexion with infantile paralysis of some or all of the muscles of the leg and foot, there is also paralysis of the quadriceps extensor muscle of the thigh, and hence, in consequence of want of power to extend the knee and hold it rigid during progression, inability to stand or walk on the affected limb. Where the paralysis of the muscles below the linee is at all extensive, there may be no distortion of the foot, the lower part of the limb being flail-like and useless; where the paralysis is less extensive, the foot may be drawn into some abnormal position by the shortenin of the muscles antagonistic to those paralysed. In either case the patient may be quite unable to stand or walk withont some form of artificial support. Or the want of power in the muscle to extend the knee has, in some cases I have seen, been compensated for by the patient placing his hand upon the lower part of the thigh, and thus forcing the knee more or less into the extended position. This manœuvre, however, necessitates a bending forward and lateral deflection of the body and theconsequent assumption of a very unsightly gait, and at times of serious curvature of the spine. The usual way of dealing with these cases is to carry double leg-irons to a calf circlet below the knee, and to continue the outer iron above the knee to a pelvic girdle, leaving a free joint at the hip, and providing the joint at the knee with a ring catch or flute-key catch. When the anterior muscles of the leg are paralysed, a toe-raising spring of some kind is also frequently employed to act on the ankle joint. By the use of the ring or flute-key catch the joint at the knee can be fixed with the limb in the extended position, thus allowing the patient, other things being equal, to stand and walls on the paralysed limb. When it is desired to flex the knee, as in sitting down or ascending or descending stairs, the ring or flute-key catch is loosened, and, the joint at the knee being no longer fixed, the leg falls into a flexed position by its own weight. Before

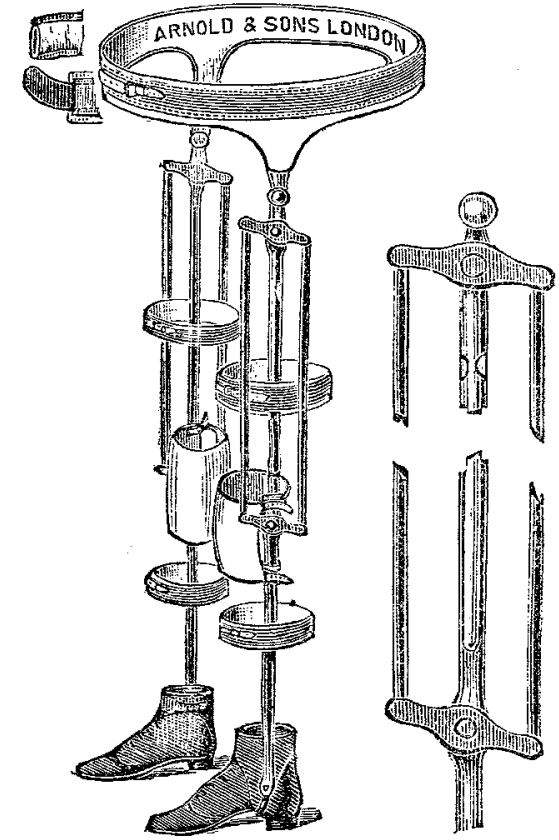

resuming the upright attitude, it is of course necessary to fix the lnee joint again, with the leg in full extension, in order that the affected $\operatorname{limb}$ may form a rigid support to transmit the weight to the ground. Now, as the extensor muscles of the leg are paralysed, this is done either by pressing the knee back with the hand on the lower third of the thigh, whilst the heel resting on the ground forms a fixed point; or by lifting the leg with the opposite foot placed behind the ankle into a line with the thigh. Then as soon as the leg is in the extended position the joint at the knee is fixed by the ring or flute-key catch. These catches, which are worked beneath the trousers or dress, are a little difficult to manage, as the leg must be fully extended before the catch can be made to fix the joint at the knee, and are consequently especially awkward to manipulate when they have been loosened for the purpose of allowing the leg to be flexed while ascending or descending the stairs. Further, as soon as the ring eatch is loosened, all voluntary control over the leg is of course lost.

Whilst considering whether something more might not be done for the relief of these cases, it occurred to me that the muscles moving the hip joint might, by a simple arrangement of levers, be made to supply the place of the paralysed extensors of the leg, and that the movements of the hip might thus be made to control, to some extent, the movements of the knee. On sitting down, the thigh is normally flexed to more or less of a right angle; whilst. in ascending or descending stairs, flexion of the hip and 
knee, but of course to a less extent (unless the step measures in depth the length of the leg when both are also Hexed at a right angle), also takes place. The instrument figured in the annexed woodcut was made by Messrs. Arnold from a rough model I supplied them for the purpose. It is so contrived that whien the hip is either flexed or extended by means of its flexor or extensor muscles, the knee, by means of the side levers (showed enlarged on the right of the figure), is also flexed or extended to a like degree. Hence, when the patient sits down, and consequently flexes his hip, the knee, by means of the levers, also becomes flexed to the same angle as the hip; and, again, in rising from the sitting posture, as the hip is straightened, so, by the same means, is the knee. As long as the hip is kept extended the joint at the knee is rendered fixed, and a rigid limb is thus provided for supporting the body. In walking, however, during the pendulum-like movement of the limb as it swings forward, while the opposite limb is on the ground, the knee should not entirely follow the movement of the hip. To allow for this a double stop-joint is further provided at the hip, permitting flexion and extension of the hip to take place to several degrees before the levers which act upon the knee are brought into action. At first it may be necessary, whilst the hip muscles are gaining power, to fix the knees completely by means of a screw nut. The instrument shown in the woodcut sufficiently explains itself, and any minute account of the mechanism seems hardly necessary. It was made for a little girl who was sent to me in the orthopæedic department with complete paralysis of the extensors and flexors of the knee on both sides, and with more or less paralysis of the left arm. She had never been able to stand on her feet, but managed to get about by walking on her flexed knees. At the present time she can walk on her feet when aided by someone taking hold of her unparalysed hand. (July 23rd: Since this was written, she can now walk alone with only one knee fixed by the screw nut.)

Weymouth-street, W.

\section{SOME FURTHER NOTES ON THE}

\section{ETIOLOGY OF ULCERATIVE STOMATITIS OR CANCRUM ORIS.}

BY ALFRED LINGARD, M.R.C.S., DIPLOMA IN PUBLIC HEALTH, CANBRIDGE.

In a previous communication I have described the occurrence of what appeared to me to be the same disease in the human subject, monkeys, and calves. The disease which I described previously affects the tongue and cheek, and secondarily the lungs, both in human subjects and in animals. A form of gangrenous pneumonia, apparently produced by similar micro-organisms, has also been observed by me in the lungs of five horses. Portions of the affected tissues inoculated in calves produced a disease resembling noma in all respects. The essential characters of the micro-organisms in all cases were similar. They consisted of long thread-like growths, the individual threads being made up of small bacilli varying in length from $0.004 \mathrm{~mm}$. or less, to $0.008 \mathrm{~mm}$. or inore, and about $0.001 \mathrm{~mm}$. in thickness. These organisms were found in oreat numbers at the line of extension of the necrotic patch. Cultivations have been made from fire cases in the human being, in one case in a monkey, in two cases in young pigs, in numerous cases in calves, and in the lungs of horses. It was then demonstrated that the inoculated disease is characterised by appearances precisely similar to those seen in the original disease. I wish here simply to enumerate the lesions found in the heart at death in the human subject, and in animals inoculated from other aninals of different species, suffering from ulcerative stomatitis or noma. The most serious conditions are those presented by rabbits after inoculation in the ear with the affected tissues from the roung pig and calf. These animals die on the tenth or eleventh day after inoculation. The ear is found to be very much increased in size from the inflammatory process, and has been found to weigh fiftyone grammes when the healthy ear only weighed twelve. As in the two cases some differences were observed at the necropsy, I will give a short account of the condition of the pericardium and heart in each.
CASE 1. Rabbit inoculated from the affected tissue of the pig. - On slitting up the pericardiun the heart was found to be enveloped by a thick, pale-coloured false membrane oneeighth of an inch in thickness, which on microscopical examination was discovered to be composed of long bacillar threads matted together. Under this membrane in the muscular substance of the heart were found yellow necrotic patches, the largest was seven-eighths of an inch in length by three-quarters of an inch in breadth, forming the apex of the heart. On section this necrosis was proved to involve the whole thickness of the left ventricular wall. The left coronary artery was distended, and gave the impression to the touch of a thick and hard piece of cord. The lungs and liver were similarly affected.

CAsE 2. Rabbit inoculutcd from the cuffected tissues of the calf.-The pericardium contained little or no fluid, but a membrane covered the surface of the heart about one-twelfth of an inch in thickness, which on microscopical examination was proved to be formed of fibrin, while numerous cells were entangled in its meshes containing large numbers of granules, also very numerous ammonio-magnesian phosphate and oxalate crystals. On removal of the membrane from the surface of the heart, several masses of yellow necrotic tissue became visible, involving the muscular structure of the apex of the left ventricle; and others were found, smaller in extent, over the upper portion of the left ventricle anteriorly and posteriorly. On section, as in the previous case, the necrosis was found to involve the whole thickness of the muscular wall, and so extend into the interior of the left ventricle. After hardening these tissues in Müller's fluid, different portions were submitted to microscopical examination. It was then found that, as in the case of the mouth ${ }^{\perp}$ so in the heart substance, the necrotic process was coextensive with the advance of a mass of bacilli.

On section of the muscular wall of the heart some small necrotic foci were observed, but there were many that could not be recognised macroscopically. These small foci, when examined with a power of 200 diameters, are found to be circular in shape. In the centre of the patch are a large number of thread-like organisms, which have rapidly increased, probably, from one or two deposited there in the first instance. Neither structure nor nuclei were to be observed in this area, owing to the nourishment having been abstracted at the expense of the cells as the bacilli gradually invaded the healthy tissue. Just outside the principal zone of bacilli there were stray or single ones between the muscle fibres, and in this situation were a few leucocytes dotted about, while still more externally was seen an extensive zone of leucocvtes surrounding the patch, which was evidently due to the inflammatory process which had started there. In many places I found these organisms in varying numbers infiltrating the intermuscular tissue and surrounding the capillaries and lymphatics. In these cases they appear like long bundles or leashes of circular contour passing along the lumen of the vessel. I also found various collections of leucocytes in the intermuscular spaces, in groups varying in size according as they contained only a number which could be counted, or as they contained so many as to make it impossible to number them. I always found one or more elements of the threads, either well-formed bacilli or round dots, which were obviously spores. In the coronary arteries and their smaller branches supplying the cardiac structures I came across vessels of different calibre, the walls of which were more or less infiltrated by bacilli. In the larger the lumen of the ressel was ocenpied by a granular substance which did not stain, evillently the remains of a blood-clot. In the centre of this clot were seen knotted skeins of threads, which readily absorbed the aniline dye. There were also seen solitary bacilli, as well as groups of the specific bacilli, in different parts of the clot in immediate contact with the intima of the vessel. The intima and elastic laminie were not invaded by these organisms; but all the rest of the arterial coat was destroyed, and consequently all definite structure lost, its place being entirely occupied by myriads of wavy threads.

In the human heart, in consequence of the early death of the patient, such serious lesions are not met with. In the five necropsies I have made on children dying of noma I have only once found heart lesion, and that was characterised by the presence of petechial spots, about from ten to twelve in number, of a dark-red colour, dotted over the surface of

${ }^{1}$ THE LAYCET, Mray, 1883; and Micro-organisms and Disease, by 